\title{
Considering Ethnicity in Teaching and Learning Statistics: Should I Worry About Where my Students Come From?
}

\author{
DARFIANA NUR ${ }^{\dagger}$ \\ dnur@maths.newcastle.edu.au \\ School of Mathematical and Physical Sciences, The University of Newcastle, Australia \\ KERRIE MENGERSEN \\ kerrie.mengersen@newcastle.edu.au \\ School of Mathematical and Physical Sciences, The University of Newcastle, Australia
}

\begin{abstract}
In the past decade there has been strong interest in the special needs of overseas students attending Australian universities, with respect to teaching and learning. This paper reports on three action research studies that address the question of whether such issues remain in the teaching and learning of statistics in particular.
\end{abstract}

Keywords: ethnicity, overseas students, teaching and learning of statistics

\section{Introduction}

This study has as its overall aim the identification of differences, if they exist, between students of different backgrounds with respect to learning statistics at two Australian universities, namely Queensland University of Technology (QUT) and The University of Newcastle. The hypothesis was that these differences might arise from four main sources: overall motivation, methods of teaching, methods of assessment and methods of learning. If such differences are detected, the question that must be addressed is what strategies may be useful for providing an equitable framework for learning in this subject. Three separate studies are reported in this paper. The first was undertaken through interviews with colleagues and students at QUT. The second is a result of teaching at the Bandung Institute of Technology in Indonesia by the first author. The third was undertaken as a formal survey of undergraduate and Masters of Business Administration (MBA) students at The University of Newcastle. In each of the three studies, the samples are neither random nor representative and, moreover, the sample size is too small to allow generalizations. However, several seminal issues were identified that were common across all studies. These would be

$\dagger$ Requests for reprints should be sent to Darfiana Nur, School of Mathematical and Physical Sciences, The University of Newcastle, Australia. 
interesting to follow up in further studies and may correlate with similar insights by other authors.

Ethnicity is a very topical social and political issue as evidenced, for example, by multinational conferences, government-funded support agencies, private community groups and political platforms concerning immigration, assimilation and Australia's place in the world. Since international students now comprise a substantial proportion of Australia's tertiary students, it is important to know whether the ethnicity of our students has potential impact on teaching and learning in tertiary education and, if so, what the main issues might be.

Australia has an image of a harmonious culturally diverse nation, committed to social cohesiveness, and this is reflected at a university level. However, it is well known that while issues such as history, entry mode, learning attitudes and skills, and personal circumstances affect all students, those with non-standard ethnic backgrounds are particularly compromised. Moreover, there are learning difficulties within a culturally homogeneous group that are exacerbated for minority groups. These include assumed knowledge, prior learning that is not recognized or utilized, exclusivity in curriculum content, lack of clarity in assessment expectations and in presentations, and implicit requirements of academic convention or cognition.

This issue has been recognized for over a decade. For example, the Quality Review Report review in a QUT memorandum (1995) concluded that internationalization of the curriculum had taken place in selected areas, but that this had yet to become a common feature of the curriculum of the institution. Moreover, realization of lifelong learning as a teaching goal was yet to be fully implemented and mechanisms were not yet in place for monitoring achievement. There was a focus on computer-based education as policy and practice, with an acknowledgment that more attention should be paid to reviewing it's educational relevance across different teaching situations. Attention to global education and meeting the needs of international students in Australia's tertiary education institutions was also been addressed in the Karpin Review (The Australian, 3rd May, 1995, pp. 24,31 ). More importantly, perhaps, it presents a damning view of formal management education: "it lacks relevance and accessibility, fails to improve performance, motivation and personal development and fails even to keep thinking up-to-date and flexible" (p. 31). It is interesting to reflect on this opinion six years after the release of this statement and in particular in the light of statistics education.

Even a decade ago, the statistical education literature on this topic was extensive and covered a diversity of issues including demographic descriptions [1], recognition of problems faced by students who are learning statis- 
tics in a foreign language [2] and comparisons of teaching and learning between countries [3], [4]. Yan [7] and Weldon and Tham [6] demonstrated that statistics courses differ between countries. However, mathematical content, statistical theory and their application also differed within the countries (China, and Indonesia and Canada, respectively). Discipline, theoretical emphasis, secondary school subjects and the intended use of statistics in further study and after graduation may all influence the statistics curriculum within a university. Universities appear to have heeded this discussion. In the authors' experience, there is increasing impetus to meet different learning needs through better design and resourcing of courses, increased access to course material through internet and other media and accommodation of off-campus and distance students. The question may now be: is the problem solved? As a university teacher, do I need to worry any more about where my students come from? This paper contributes to this discussion.

\section{A QUT Experience}

The first study reported here was undertaken at QUT. It comprised a focus group drawn from undergraduate classes taken by the second author in 1995. Students were informed that the aim of the discussion was to discuss whether where students came from should influence teaching and learning at QUT, and if so, how. A profile of the 15 students interviewed is as follows: twelve were less than 25 years of age; country of residence was Australia (6), Papua New Guinea (1), USA (1), China (3), Hong Kong (2), Korea (2); university year was first (10), second (3), third (2); major course of study was physical science (1), biological science (8), information technology (4), statistics (2).

As part of the discussion, members of the focus group were invited to ask questions considered relevant to a discussion of ethnicity in the content and conduct of their statistics courses at QUT. Interestingly, statistics per se was not the major factor identified in these questions, despite its agreed difficulty. Instead, the questions on which agreement was reached were focused on issues that impacted more widely on teaching and learning. The questions and the resultant themes were as follows.

What are the major difficulties you face in learning statistics at QUT?

All students, regardless of age or cultural background, expressed concern about the amount of material, unfamiliarity with the technical language and software, and the relevance of the subject. More than half of the students wished for access to resources other than the specified texts and 
handout materials. Although they had been encouraged to 'use the library', they wanted more specific directions to relevant material. Three students (two older, one non-Australian by birth) discussed difficulties in studying due to family commitments. No differentiation was made between teaching and learning difficulties despite some gentle prompting.

What is the major difference between teaching and learning at your previous educational facility and at QUT?

For twelve students the last academic institution was secondary school, although five had worked between school and university. All students expressed the opinion that at school there was more 'spoonfeeding' of mathematics with more constant supervision by teachers and greater requirements to complete work. The freedom that university afforded was suggested to be a two-edged sword in statistics, especially since so much of the learning is cumulative. Two of the overseas students (from India and Malaysia) planned to return to their home countries to teach. Both found that there was a greater emphasis in the Australian courses on individual work and less direct support for students by lecturers than in their own countries.

Are you comfortable with giving verbal presentations in class?

Although all students liked hearing presentations by other students, only one volunteered that he liked to give them! All but one Australian had given some form of formal oral presentation at school if not at university; of the non-Australian students only one had ever been required to do this!

What is your opinion of assessment based on assignments, practical projects and exams?

Answers here were surprisingly clearcut. Students from Australia and the USA preferred continuing assessment and enjoyed practical projects despite their time-consuming nature. The students from India and Asia preferred exams and straightforward assignments, commenting almost unanimously that they were not sure what was required in a practical project and that they found collecting data for a practical project was a daunting task. The time factor with all continuous assessment was a major discussion point for mature-aged students and those in second and third years of study. All but two students agreed that it was easier to work in groups on assignments and projects, although all first year students and some later-year students commented that they did not know people well enough to form groups early in the semester when it would have very valuable. One student from Asia suggested that Asians should not be grouped entirely together, although she admitted that she felt intimidated in a group of Australians. 
How would you rate the usefulness of the following items in terms of learning, on a scale from 1 (very poor) to 5 (very good)? Technical assignment, exploratory/self- directed assignment, practical assignment, tutorial worksheets, lectures. (Sample pieces of these assessment were provided for respondents after this question was identified.)

Based on average ratings, the items were rated in the following order (mean and standard error of mean are given in brackets): technical assignment $(2.6,0.23)$, practical assignment $(2.9,0.28)$, lectures $(3.0,0.19)$, tutorial worksheets $(3.3,0.19)$, exploratory/self directed assignment $(3.6,0.17)$. With one exception there was no significant difference in average ratings between students based on age, country of residence or degree major. The largest difference was between age groups for all items except lectures. Older students rated the practical assignment significantly higher than did younger students. The largest difference between ethnic groups was seen in the rating awarded to lectures, with these being more highly regarded by students with overseas residence.

Informal discussion was held in the focus group about relevant issues in learning that were a consequence of cultural background. The participant from Papua New Guinea explained that major cultural adjustments were faced by the majority of students from this country. He identified 'us versus me' societies, describing his home 'wan tok' system in which things are undertaken together at all levels of society including school activities and assessment. In contrast, the Australian culture in general and the university system in particular was perceived as an individual framework. He also identified 'missing links' in language and thinking; differences in cultural beliefs and practices that required commitments that were still not fully recognized; passive versus active approaches to learning, where the impetus in the Australian culture to find information rather than be given it whereas in Papua New Guinea it was common to receive all materials required for study with detailed support from the teacher; an impetus to 'read, read, read'; difficulties with assessment that involved 'industry projects' in which applications may be 'foreign', leading to a double workload for overseas students; family commitments at home, with expectations that income could be generated in Australia for support of family and related activities. Lastly the speaker raised the need to balance the changes in outlook and culture that an Australian education afforded with the reality of professional, social and personal life on his return home. A woman whose place of residence was Australia but whose place of birth and secondary education was Korea then spoke. She was a second year student in banking and finance. Despite her greater exposure to Australian culture, she still found difficulties with language and culture in her university 
studies. She identified in particular the 'openness' of people in Australia and differences in train of thought and interpretations of jokes, especially those along commercial, political or sarcastic lines. Social difficulties, including different views of the roles of dancing and drinking at parties were also raised as impacting on general integration and academic performance. An important issue that she raised was the differences in 'cues' given to enable younger people to talk to older people, females to talk to males, and so on. Also, small talk by lecturers about a student's home country was sometimes taken to be offensive in that it was seen to be an attempt to put down a foreign student or ostracize them. The group generally agreed with her perception that there was a difference in outlooks between students who were in Australia temporarily and those who were here on a more permanent basis.

\section{A Newcastle Experience}

As part of a statistics course with MBA students at the University of Newcastle in 2001, a study focused on overseas students' stress was undertaken in collaboration with student Lin Zhang. In discussion with class colleagues, Lin identified stress, the response variable, as the feeling of being in a challenging or threatening situation, and measured this on a scale from 1 to 7 . Identified explanatory variables included gender, length of time in Australia, employment (whether the student also has a job), sleeping (reported ability to sleep well), type of accommodation (student residences, off-campus), perceived difference in lifestyle and perceived difference in study style. This last variable, study style, was described as the way in which courses are designed, delivered and assessed at this university. Note that, as in the QUT study described in Section 2, these issues again do not directly target statistics per se but instead the more general issue of learning at an Australian university.

A convenience sample of 35 students drawn from undergraduate friends of the MBA students completed a survey of 12 questions targeted at the above hypotheses. Issues such as bias and nonrepresentativeness associated with such a sample were discussed with the class but are not reported here. The following results were reported. Students felt in a challenging or threatening situation because of the new lifestyle (89\%) and because of the different study style (80\%). The most important variable of those examined in reducing students' stress was the length of time spent in Australia. Sleeping, study style and employment were also strongly associated with the response on stress. There was no substantial difference between responses for males and females. The variables considered explained about 
$40 \%$ of the variation in responses to stress. Other issues contributing to overseas students' stress are clearly influential.

Clearly, even now, overseas study puts students in a perceived challenging or threatening situation. Interestingly, although such feelings are common, the survey also revealed that students' attitudes to the new situation were generally positive. All students declared that they would not give up study as a result of these feelings.

\section{An Indonesian Experience}

The third study comprised an in-depth examination of differences between teaching and learning in Australia and one of our nearest neighbors, Indonesia. The University of Newcastle has a growing proportion of Indonesian students at both undergraduate and graduate level, yet most lecturers are not aware of the Indonesian secondary or tertiary system of teaching and learning. The first author is uniquely placed to contribute to this story, since she has taught at universities in both countries for many years and was an overseas student herself at an Australian university for four years. Based on personal experience and discussion with Indonesian students at The University of Newcastle, the following points emerged.

The major difficulties in learning statistics in Australia is how to raise questions in the class with confidence as an active student. Although this could be explained by a language barrier, a more pervasive explanation is the different expectations of a lecture. Indonesian students are generally used to a passive culture in which lectures are used for taking notes and understanding of the material occurs afterwards.

The teaching and learning system in Indonesia is quite different to Australia at all levels, from secondary to tertiary. At secondary school the curriculum in Indonesia is considerably heavier with around 10-15 subjects in one term. These are delivered with a one-way direction of teaching: the teacher explains the material; discussion is rarely afforded, and spoonfeeding is common. These practices are reflected in the tertiary system, with undergraduate courses typically completed in eight semesters (140 credit points) with each subject contributing between 2 and 4 credit points. Students are given much more material in a more passive learning environment.

Giving verbal presentations in the class in Indonesia at secondary level is not usual. At tertiary level, this may be encouraged in some subjects, but it is rare.

In Indonesia, assessment at secondary school is based on much homework and one final test in each term. At tertiary level, assessment may be based 
on one mid- semester test and one final exam. Some lecturers may give assignments, but the weight given to the final exam is quite large.

\section{Discussion}

Four issues have emerged clearly as a result of these studies.

First, different (perhaps distinguishable groups of) students react differently to various methods of teaching. Some students enjoy active oral presentation and involvement in class, but this may be related to ethnicity, age, discipline, learning style or other individual factors. Although presentation skills are perceived as useful for general personal and professional development, there is a need for careful facilitation of these activities and perhaps an avenue for alternative methods of assessment.

Although some differences in attitudes and learning styles may be attributed to cultural diversity, there is also wide diversity among culturally homogeneous groups, with differentiation arising through age, discipline and personality. This implies that while there must be an acknowledgment of differences in needs based on ethnicity, the temptation to globally categorize students on such a basis must be resisted. As a teacher, it is important to structure the learning environment so that the recognition of differences arising from ethnic diversity is a liberation rather than a limiting generalization.

Importantly and interestingly, all three studies did not raise issues regarding the impact of ethnicity in learning that were specific to statistics. Difficulties identified in learning statistics, including the nature and volume of material, were uniformly acknowledged. Issues of assessment, presentation and learning culture were common to all courses, not specifically statistics. The implication of these observations may be taken to be positive or negative. From a positive perspective, it might indicate that statistics presents no extra burden of learning and that broad strategies to accommodate differences in learning will suffice to enhance learning in statistics. From a less positive perspective, it may be that the fundamental difficulties in learning statistics overwhelm any differences arising from ethnicity and it is only when these are overcome that the latter issues will emerge. These studies did not to assess which of these interpretations is valid but the issue points to a direction for further research.

The third point arises from the study of overseas students at The University of Newcastle. The collaborator in this study, Lin, made a final conclusion that since overseas students experienced stress but were generally positive about the overseas experience, 'overseas students' stress is normal'. Although this interpretation may be debated, in any event it is 
not grounds for complacency among university teachers. Indeed, it appears that although progress has been made in the last decade, there is still much work to do to improve teaching and learning for overseas students.

The last point is that it behoves all educators to learn about the different education practices in those countries from which our students are drawn. Even among close neighbors such as Indonesia and Australia, there is stark contrast in teaching and learning statistics at secondary and university level. Increased awareness of these differences cannot fail to be translated in at least subtle ways in the practice of teaching and learning.

The conclusions reached in this paper are based on small focus groups and selected institutions. The results are not claimed to be strongly representative of the Australian tertiary student community and the reporting of the results is subject to personal interpretation by the authors. However, it is hoped that the issues identified provide cause for reflection and consolidation.

\section{Acknowledgments}

We wish to thank all of the participants in the studies reported in this paper.

\section{References}

1. M. Anderson and S.E. Fienberg. Black, white, and shades of gray (and brown and yellow). Chance, 8(1):15-18, 1995.

2. R. Hubbard. Teaching statistics to students who are learning in a foreign language. Proceedings of ICOTS 3, Editor D. Vere-Jones, ISI Publication, 514-517, 1991.

3. B. Kaur. She teaches statistics in Singapore schools. Proceedings of ICOTS 3, Editor D. Vere-Jones, ISI Publication, 517-520, 1991.

4. D. Ladiray. A school of statistics inside a government statistical office ENSAE, a French example. Proceedings of ICOTS 3, Editor D. Vere-Jones, ISI Publication, 522-527, 1991.

5. Report on Quality Review of Teaching and Learning. QUT Internal Memorandum from the Vice-Chancellor, March 31, 1995.

6. L. Weldon and P. Tham. What is basic statistics? Lessons from a CanadianIndonesian project. Proceedings of ICOTS 3, Editor D. Vere-Jones, ISI Publication, 95-99, 1991.

7. S.J. Yan. Probability and statistics courses in the universities in China. Proceedings of ICOTS 3, Editor D. Vere-Jones, ISI Publication, 91-94, 1991. 


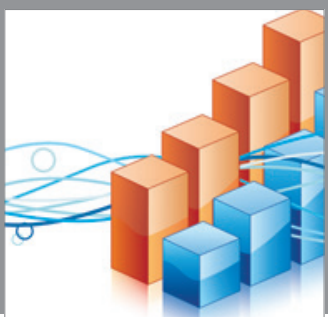

Advances in

Operations Research

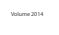

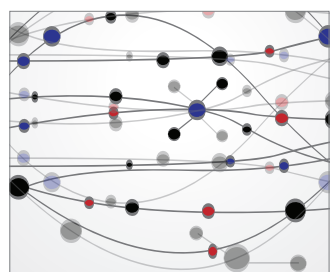

\section{The Scientific} World Journal
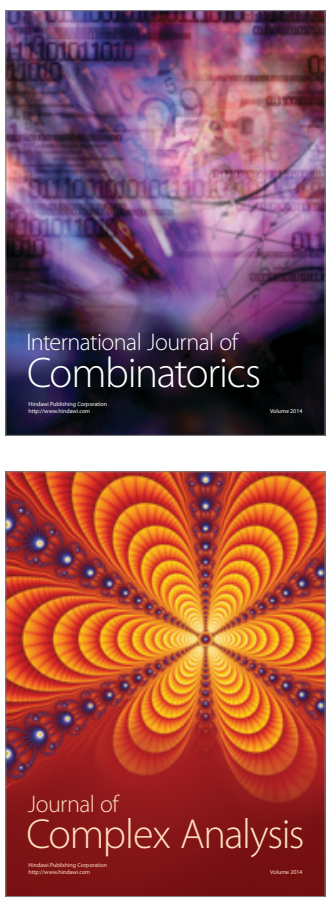

International Journal of

Mathematics and

Mathematical

Sciences
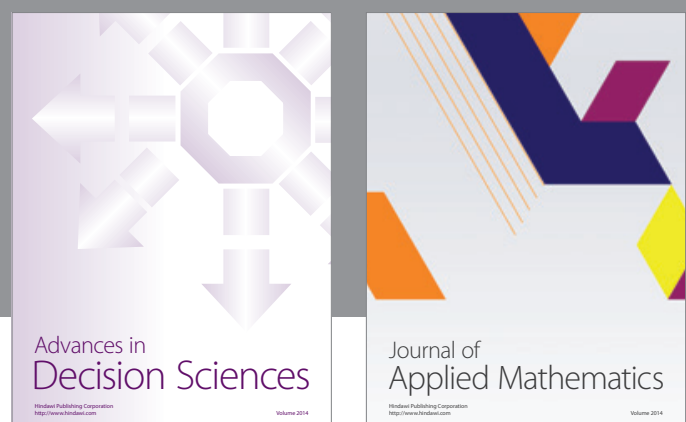

Journal of

Applied Mathematics
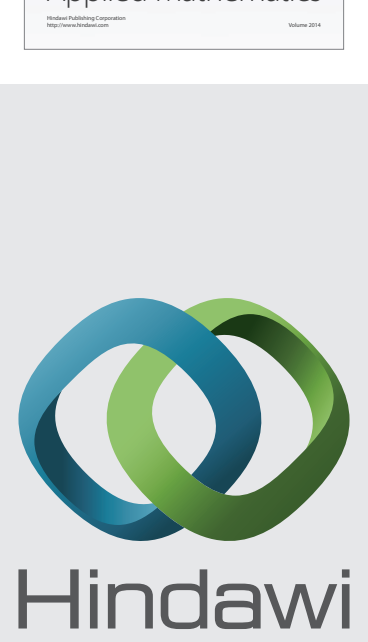

Submit your manuscripts at http://www.hindawi.com
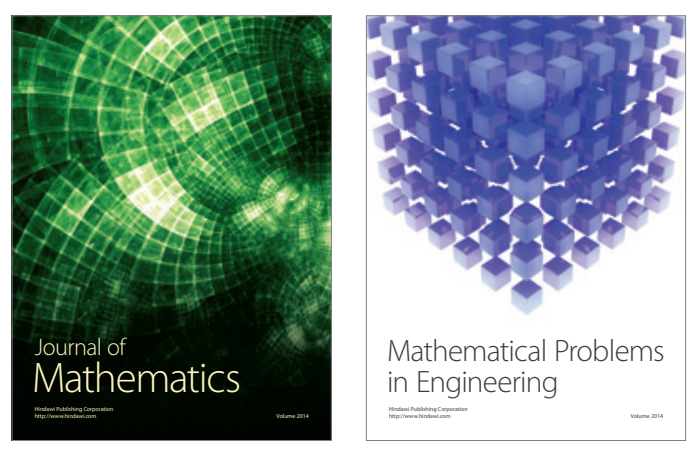

Mathematical Problems in Engineering
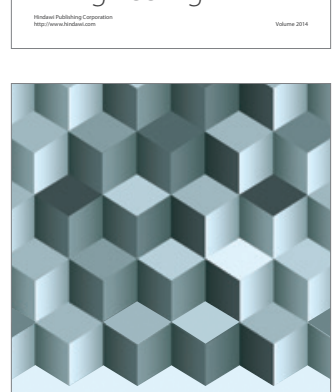

Journal of

Function Spaces
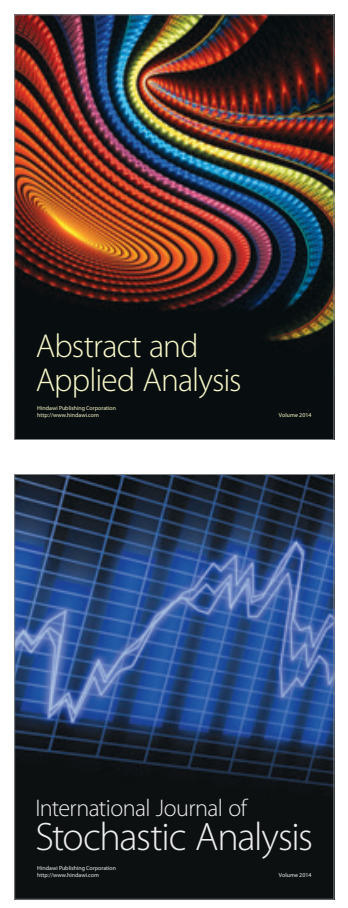

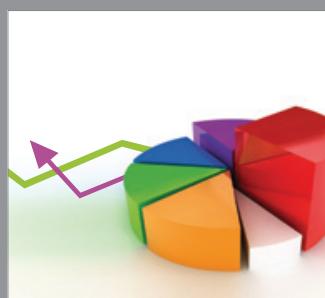

ournal of

Probability and Statistics

Promensencen
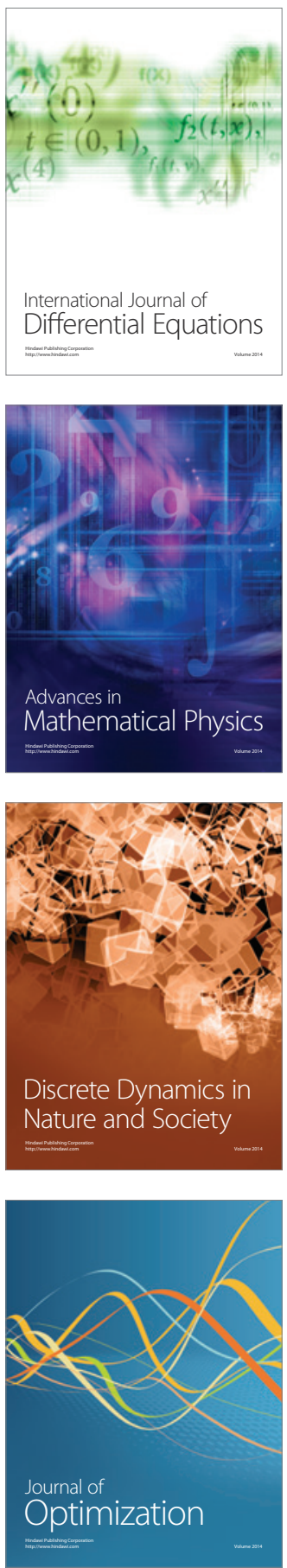